\title{
New Pedestrian Model Based on a Social Force Model with Sub-goals
}

\author{
Tohru Mizuno, Hirohide Haga* \\ Graduate School of Science and Engineering, Doshisha University, Japan. \\ * Corresponding author. Tel.: +81-774-65-6978; email: hhaga@mail.doshisha.ac.jp \\ Manuscript submitted September 12, 2019; accepted November 10, 2019. \\ doi: $10.17706 /$ jcp.14.11.634-649
}

\begin{abstract}
This article proposes a new pedestrian model that precisely simulates the avoidance behavior so as to realize the pedestrians' walking action. For the design of the facility, the simulation is used as a means for predicting and analyzing the movement of pedestrians. However, most of the simulators focus on the flow of pedestrian but do not consider the movement of each pedestrian. As a result, conventional simulators are useful to simulate the phenomenon of macro level movement of pedestrian but are not useful to simulate the micro-level simulation. Therefore, we aim to implement the pedestrian flow simulator that enables us to observe each pedestrian behavior by using the Multi-Agent System. Research on pedestrian model began in the 1970s, but all models cannot simulate the behavior of the pedestrian individual well. Social Force Model, which is one of the typical pedestrian models, reproduces the behavior using the equation of motion by considering the mass point pedestrian. Accordingly, this model is effective in high-density state. By contrast, there is several problems in low-density state: the avoidance speed and trajectory. Hence, we propose a new model by introducing the concept of sub-goal and new force in order to solve this problem. Furthermore, the evaluation experiment shows that proposal model improves these problems.
\end{abstract}

Key words: Multi agent simulation, pedestrian model, social force model, sub-goal.

\section{Introduction}

The purpose of this study is to propose and verify a simulation model that faithfully reproduces pedestrian avoidance behavior with a view to implementing a simulator that embodies various characteristics of real pedestrians.

Although pedestrian models have been studied since the 1970s, none of them can be said to adequately reproduce the behavior of individual pedestrians. Existing models of the behavior of individual pedestrians include cellular automaton models and virtual force models. A typical example of a cellular automaton model is the ASPF model developed by Toshiyuki Kaneda [1]. This model reproduces crowding phenomena at scramble intersections and commercial facilities by describing behavior based on 21 action rules. However, since this model considers a partitioned space, it limits the range of movements that can be expressed and is unable to reproduce the finer movements

of pedestrians. On the other hand, models that assume virtual forces include Helbing et al.'s Social Force Model (hereinafter abbreviated as SFM) [2]. This model treats pedestrians as point masses and uses equations of motion to represent their behavior, allowing it to reproduce the characteristics of pedestrian groups in high-density conditions [3]. However, the individual pedestrians in this model are unable to avoid obstacles that are placed in their path, and when they do start to avoid objects, their distance from these 
objects is less than what is observed in practice. The paths they follow after avoiding an object are also unnatural. Another problem is that pedestrians are unable to avoid one other under certain conditions when their paths cross.

Therefore, in this article we propose a new model to resolve the issues of SFM. In Section 2 we describe SFM and its issues, and then in Section 3 we propose our new model. In Section 4, we describe a simulator that implements this method, and we present the results of evaluation tests performed using this simulator. These results are discussed in Section 5.

\section{Pedestrian Models}

\subsection{Previous Studies of Pedestrian Models}

The pedestrian models that have been proposed so far can be divided into micro-models that reproduce the behavior of individual pedestrians, and macro-models that aim to reproduce the overall flow of pedestrians. A variety of methods have been proposed for both model types. The leading pedestrian models are as follows:

1. Network model (macro-model): The environment between a pedestrian's current location and destination is visualized in the form of a lattice-shaped pathway network, where rules that embodying the behavior of pedestrians are consulted in order to model their choice of path. Since a characteristic of this model is that the pedestrians have to move on the pathway network, it is not possible to reproduce detailed pedestrian movements.

2. Fluid model (macro-model): The flow of pedestrians is treated as a continuous fluid, and the flow of traffic is represented using equations of motion and continuity. The characteristics of this model mean that it is unable to reproduce the movements of individual pedestrians but can reproduce the overall behavior of traffic flow.

3. Cellular automaton model (micro-model): In this model, a space is represented by a number of cells. The movements at the next step are determined by consulting rules of movement based on the state of the surrounding cells. This model is easy to implement because the rules are simple, but it seems to produce results that are unnatural at the level of detailed human actions, so it cannot really be said to accurately reflect reality.

4. Virtual force model (micro-model): This model represents pedestrians as being acted upon by virtual forces like electricity or magnetism. In this model, the path selections and other behaviors of pedestrians are not a reflection of their intentions, but their movements can be reproduced by considering the forces acting on pedestrians and obstacles.

\subsection{Social Force Model (SFM)}

The social force model (SFM) belongs to category (4) in 2.1 and was proposed by Helbing et al. [2]. It has been shown that this model can reproduce situations with a high density of pedestrians [3]\}. An overview of SFM is presented here.

\subsubsection{The three virtual forces of SFM}

A pedestrian is regarded as a point mass $m$. The movements of pedestrians are determined by solving the following motion equation (1) for each point mass to reproduce their accelerations and positions for the next step.

$$
m \frac{d v}{d t}=F
$$

Furthermore, each point mass is assumed to be acted on by three forces.

1. Attraction towards the destination: In general, a pedestrian will take the shortest route to a destination. To represent this movement, Equation (2) represents the forces acting on a pedestrian in terms of the direction vector $(\overrightarrow{\mathrm{e}})$ from the current location to the destination, the pedestrian's ideal 
walking speed $(\boldsymbol{V})$, and the pedestrian's current walking speed $(\vec{v})$. Here, $\tau$ is a characteristic parameter of the pedestrian. A smaller value of $\tau$ means that the force acting towards the destination is larger, and a smaller value of $\tau$ means that the other forces acting on the pedestrian have larger effects, thus making the pedestrian more susceptible to effects from the environment.

$$
F^{g}=\frac{\mathrm{Ve}(\mathrm{t})-\overrightarrow{\mathrm{v}}(\mathrm{t})}{\tau}
$$

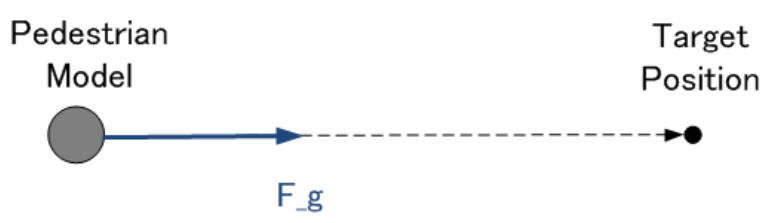

Fig. 1. Force acting towards the destination.

2. Repulsion from obstacles: Pedestrians have to avoid obstacles. They are not only subjected to physical forces when they come into contact with obstacles, but they also feel psychological pressure from obstacles when they approach within a certain distance. The force that expresses this characteristic is represented by Equation (3) using a vector ( $\vec{r}$ ) from the pedestrian to the obstacle, and parameters $U$ and $R$, which are inherent to the obstacle. The $U$ parameter is the maximum value of an obstacle's effect on pedestrians, and the $R$ parameter expresses the range of this obstacle's influence.

$$
f^{O}=-\frac{\vec{r}}{\|\vec{r}\|} U e^{-\| \vec{r} \mid / R}
$$

3. Repulsion from other pedestrians: Pedestrians also have to avoid bumping into other people. As with obstacles, a pedestrian feels psychological pressure when approaching close to another person and will thus act to maintain a certain distance. Therefore, the forces whereby a pedestrian avoids another person are expressed by two equations (4) and (5) using the direction vector $(\vec{r})$ from the pedestrian to the other person and the direction vector $(\vec{e})$ from the current location to the destination. Here, $\vec{v}$ represents the other person's walking speed, and $\vec{v} \Delta$ t represents the distance moved by the other person in one step.

$$
\begin{gathered}
f^{S}=-\frac{\overrightarrow{\mathrm{r}}}{|| \overrightarrow{\mathrm{r}}|| \mathrm{Ae}^{-\mathrm{b} / \sigma}} \\
2 b=\sqrt{|| \overrightarrow{\mathrm{r}}+|| \overrightarrow{\mathrm{r}}-\mathrm{v} \Delta \mathrm{t} \overrightarrow{\mathrm{e}} \|)^{2}-(\overrightarrow{\mathrm{v}} \Delta \mathrm{t})^{2}}
\end{gathered}
$$

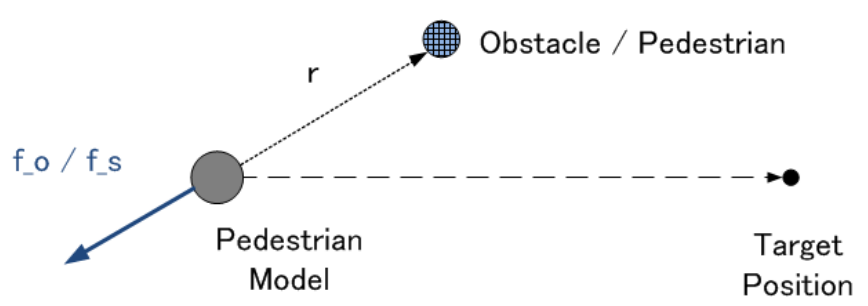

Fig. 2. Obstacle avoidance forces.

\subsubsection{Settings field of view}

In SFM, the magnitudes of repulsive forces are adjusted by setting the model's field of view. Specifically, the effects of repulsive forces received from obstacles or other people that are outside the model's field of view are mitigated using a coefficient $c(0<c<1)$. Here, $\varphi$ represents the pedestrian's field of view angle, and $\theta$ represents the angle between the direction of travel and the direction of the obstacle. 


$$
F= \begin{cases}f & (\phi \geq \theta) \\ c f & (\phi<\theta)\end{cases}
$$

\subsection{Verification of SFM of Avoidance Action}

In general, pedestrian avoidance actions involve the selection of both a path and a speed. In some situations, it is conceivable that only one of these may be selected, while in other situations both may be used. Previous studies of avoidance action with a focus on path selection include those of Tatebe et al. [4] and Yoda et al. [5].

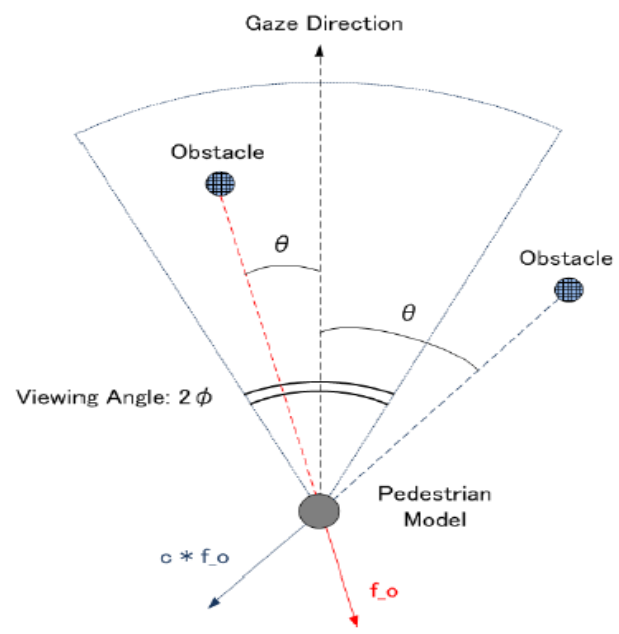

Fig. 3. Adjustment of repulsive force according to _eld of view.

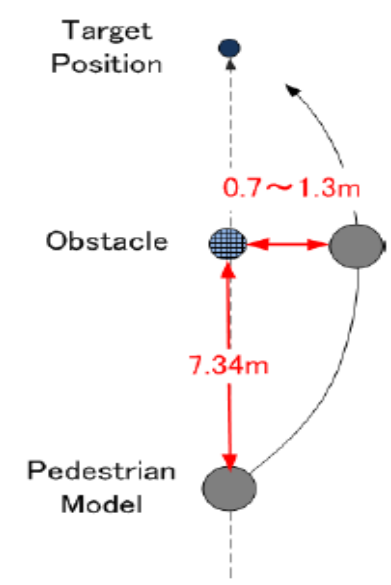

Fig. 4. Avoidance by path selection.

Tatebe et al. studied how pedestrians avoid stationary obstacles, and reported that, based on their measurements, the distance to an obstacle at which a pedestrian starts to take evasive action is related to the obstacle's size and direction. They concluded that the average distance was $7.34 \mathrm{~m}$ for an obstacle of about the same size as a human. Yoda et al. performed tests of how pedestrians avoid people who are walking the other way and reported that a pedestrian's speed during an avoidance maneuver remains constant irrespective of the avoidance path. They also reported that the average distance of two pedestrians passing each other is $0.7-1.3 \mathrm{~m}$. On the other hand, there have hardly been any studies of avoidance actions involving changes of speed. Yamamoto et al. [6] measured the movement of pedestrians on intersecting paths while being aware of other people and reported the results of measuring the characteristics of this behavior. They 
reported that when a pedestrian decelerates to avoid another person, the speed decreases sharply to $0.8 \mathrm{~m} / \mathrm{s}$ at $1.2 \mathrm{~s}$ after the situation shown in Fig. 5, the distance between them decreases to $1.5 \mathrm{~m}$ as shown in Fig. 6, and then after $0.9 s$ the pedestrian accelerates back to the original speed. The SFM avoidance behavior was verified based on this data.

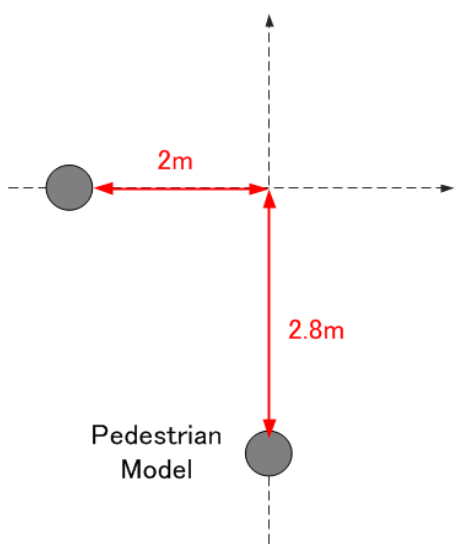

Fig. 5. Avoidance by speed selection (at start deceleration).

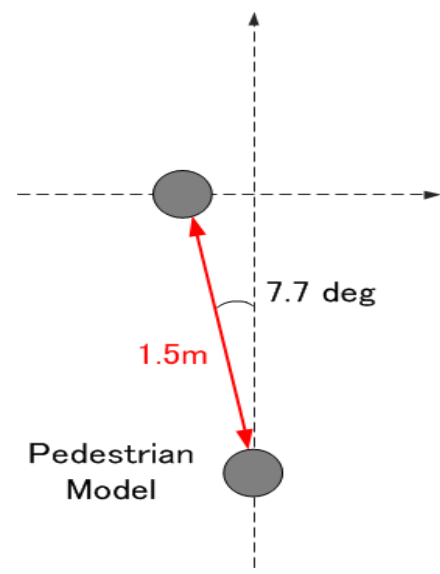

Fig. 6. Avoidance by speed selection (at the end of deceleration).

\subsubsection{Avoidance of obstacles}

A walking simulation is performed in a planar region $20 \mathrm{~m}$ long and $4 \mathrm{~m}$ wide, with a pedestrian situated at the lower end and the destination at the upper end. An obstacle with a diameter of $0.2 \mathrm{~m}$ is placed at the center of the planar region, and the pedestrian's changes of speed and avoidance path when passing this obstacle are verified. To verify the avoidance path, the pedestrian's initial speed and target speed are assumed to be $v_{0}=1.35 \mathrm{~m} / \mathrm{s}$ and $v=1.35 \mathrm{~m} / \mathrm{s}$, respectively. The pedestrian's initial position was chosen so as to simulate two scenarios:

(a) avoiding an obstacle on the walking path $(x=0)$, and

(b) avoiding an obstacle close to the walking path $(x=0.3)$

Also, changes of speed were verified by setting the initial speed to $v_{0}=0 \mathrm{~m} / \mathrm{s}$ or $v_{0}=1.35 \mathrm{~m} / \mathrm{s}$ at the starting position in case (b). Fig. 7. shows the positional relationship between the obstacle and the pedestrian, and the external forces acting between them. With reference to the study by Helbing et al., the pedestrian parameters were set to $m=60$ and $\tau=0.1$, and the obstacle parameters were set to $U=10$ and $R=0.2$. The simulated walking path and speed changes of the pedestrian are shown in Fig. 8 and 9, respectively. 
(a)

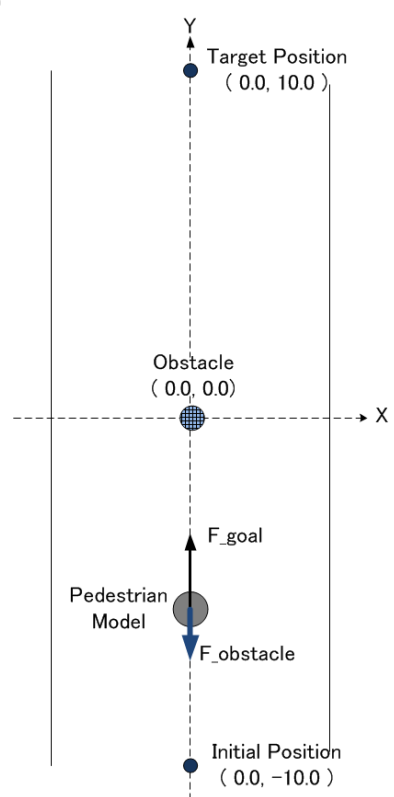

(b)

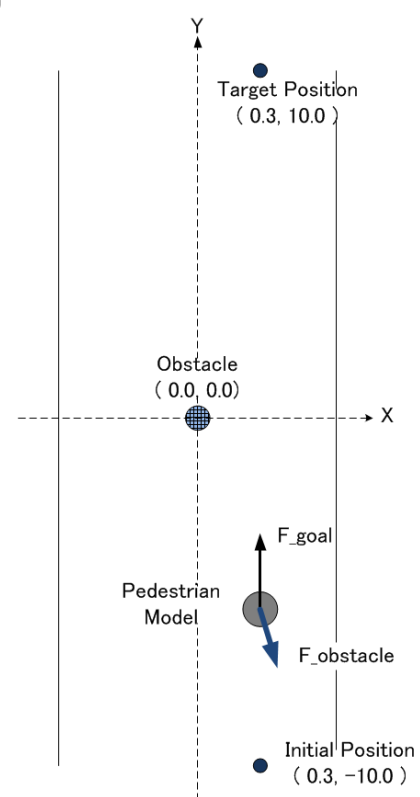

Fig. 7. Positional relationship and external forces between obstacle and pedestrian.

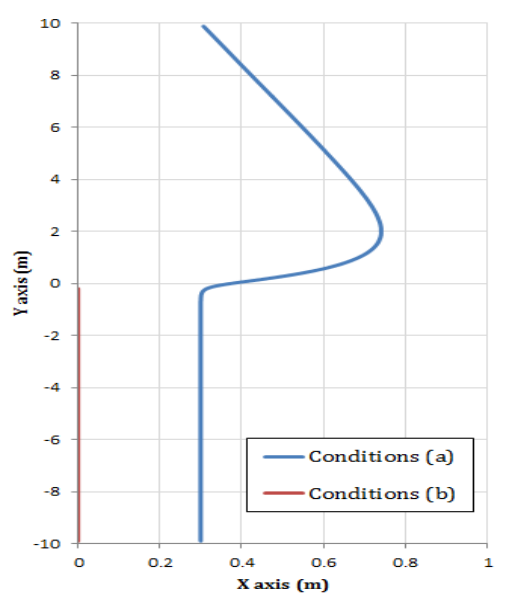

Fig. 8. Avoidance paths.

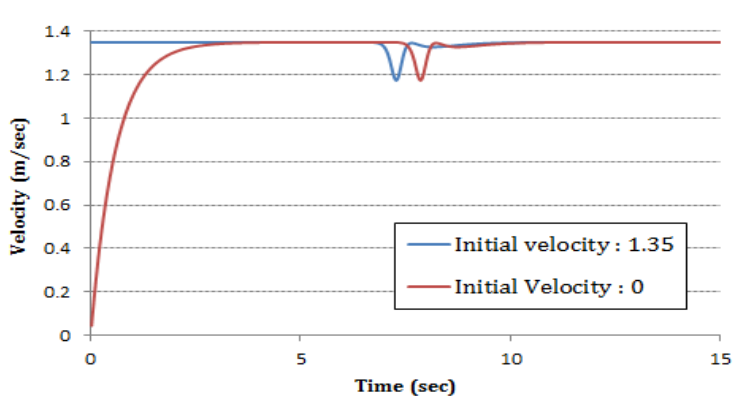

Fig. 9. Speed variation.

As Fig. 8 shows, the pedestrian was unable to avoid the obstacle in case (a) and became stuck. This is because at the starting position, as shown in Fig. 7, the force $F_{o}$ acting on the pedestrian from the obstacle is collinear with the force $F_{g}$ from the pedestrian's destination, so there is no external force acting in the $\mathrm{x}$ direction. On the other hand, Fig. 8 also shows that it was possible to avoid the obstacle at $x=0$ in case (b). However, the pedestrian's distance from the obstacle at the start of avoidance was just $0.38 \mathrm{~m}$, which is much 
smaller than the value of $7.34 \mathrm{~m}$ reported in the literature. Furthermore, the pedestrian's distance from the obstacle during avoidance was $0.17 \mathrm{~m}$, which is again unrepresentative of the reported figure of $0.7-1.3 \mathrm{~m}$. Focusing on the avoidance path, the maximum value of the $\mathrm{x}$ coordinate reaches $x=0.74 \mathrm{~m}$ at $y=2.1 \mathrm{~m}$ after passing the obstacle, so it is inferred that the avoidance action continues even after the obstacle has been passed. Looking at the changes of speed in Fig. 9, it can be seen that the speed during avoidance decreases by $17 \%$ regardless of the initial speed, which conflicts with earlier studies in which the speed remained constant during avoidance.

As the above results show, it is not possible to avoid the obstacle in case (a), while in case (b) although some improvement can be expected by adjusting the parameters, the start of the avoidance action is too late, the avoidance path is unnatural, and the change of speed is too large.

\subsubsection{Avoidance of people on interesting paths}

Pedestrians were placed at the left and bottom of a planar area, and their respective destinations were set at the right and top of the area so as to make their walking paths intersect. We then verified the speed variations of their avoidance actions under these conditions. Fig. 10 shows the positional relationships and external forces during the verification experiment. The pedestrians were given an initial speed of $v_{0}=1.35 \mathrm{~m} / \mathrm{s}$ and a target speed of $v=0 \mathrm{~m} / \mathrm{s}$, and the parameters governing the repulsive force between the pedestrians were set to either:

(a) the values used by Helbing et al. $(A=2.1, \sigma=0.3)$, or

(b) values that produced a stronger repulsive force $(A=18, \sigma=8)$.

With the other parameters set to $m=60$ and $\tau=0.1$, the simulated walking path results were as shown in Fig. 11.

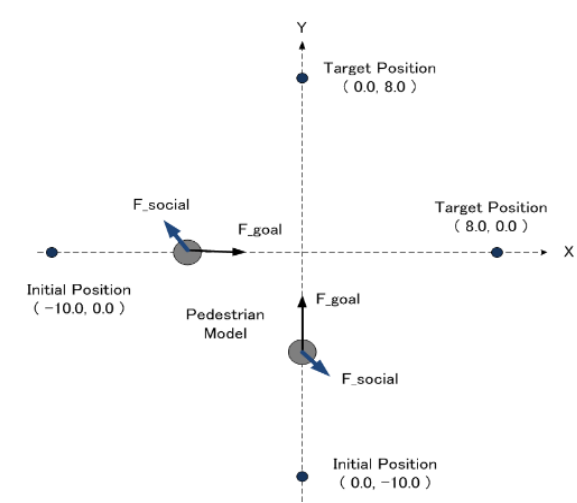

Fig. 10. Experimental conditions.

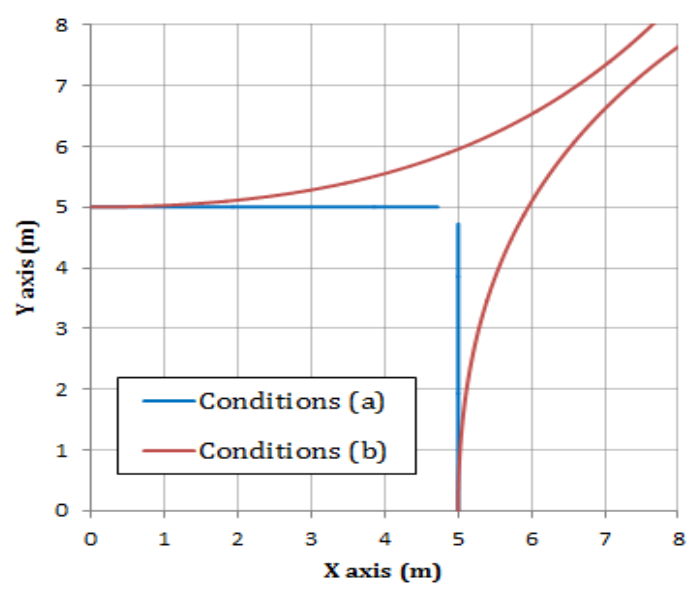

Fig. 11. Experimental results. 


\subsection{Some Issues of SFM}

From the results of these verification experiments, we can say that the model has the following issues:

1. Avoiding obstacles:

- Pedestrians cannot avoid objects that are on their walking path.

- Obstacle avoidance does not start until the pedestrian is close to the obstacle.

- Pedestrians move too close to obstacles that they are avoiding.

- The obstacle avoidance distance reaches a maximum after the obstacle has been avoided.

- The pedestrian's speed decreases when avoiding obstacles

2. Avoiding other pedestrians:

- Under certain conditions, other pedestrians cannot be avoided.

- Both pedestrians move in the same way.

A major factor behind the issues with obstacle avoidance is the fact that it is expressed solely in terms of a repulsive force from the obstacle. Consequently, depending on the directions of the repulsive force and $F_{g}$, the pedestrian can slow down and may even get stuck. Also, the fact that the maximum avoidance distance occurs after the obstacle has been avoided is thought to result from the effects of acceleration due to the model being based on equations of motion. Similarly, when two pedestrians try to avoid each other, they both behave in the same way because their actions are based solely on the repulsive force from the other pedestrian.

\section{Proposed Method}

\subsection{Introducing the Concept of "Sub-goal"}

Based on the SFM verification experiments discussed in Section 2, it can be inferred that repulsive forces alone are not sufficient for implementing avoidance action. To solve this issue, we propose a model that sets new sub-goals in order to avoid obstacles. A sub-goal is a point through which the pedestrian should pass in order to reach the destination. A pedestrian model that uses sub-goals was previously proposed by Yanagisawa et al. [7]. In their model, a sub-goal is set when there is an obstacle in the pedestrian's path, so that the obstacle can be avoided. However, this model is inadequate in that it cannot be used when avoiding obstacles that are close to the walking path, and in that the variation of speed during avoidance has not been verified.

In the model we propose in this article, we use the angle $\alpha$ and distance $r$ to identify an obstacle that lies on or near the walking path. The angle $\alpha$ represents the angle from the direction of travel and adjusts the range of objects that are regarded as obstacles. Also, $r$ represents the distance from the pedestrian to the obstacle.
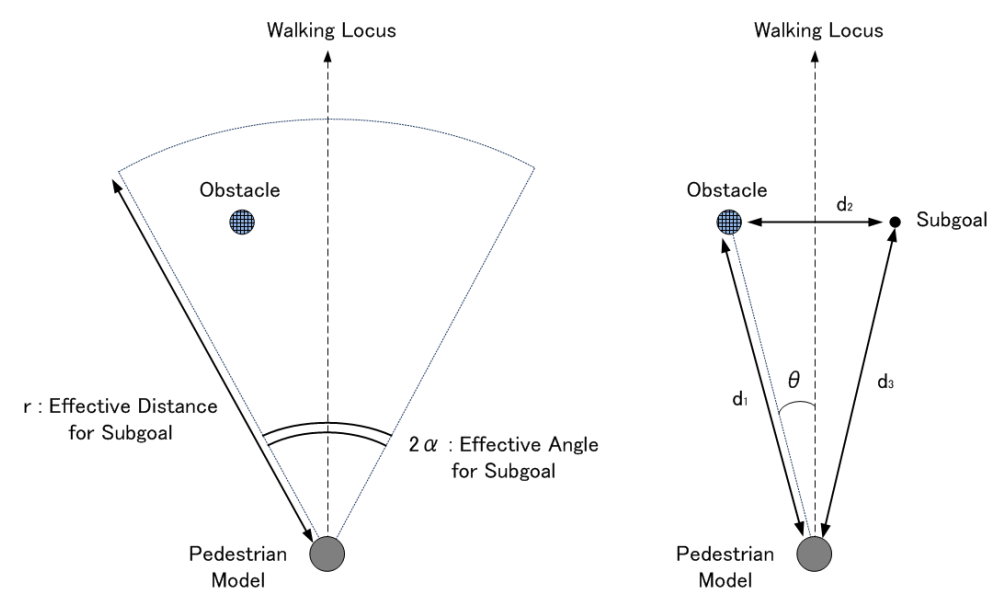

Fig. 12. Sub-goal parameter settings. 
The sub-goal settings are made using four parameters $d_{1}, d_{2}, d_{3}$ and $\theta$. Parameter $d_{1}$ is the distance between the obstacle and the pedestrian, and $\theta$ is the angle between the direction of travel and the direction of the obstacle (positive in the clockwise direction). These two parameters are used to prioritize objects when there are two or more objects regarded as obstacles. Parameter $d_{2}$ represents the distance between the obstacle and the sub-goal. Also, considering the acceleration effect that was mentioned as one of the issues with SFM, parameter $d_{3}$ represents the distance from the sub-goal beyond which the pedestrian's goal is switched from the sub-goal back to the original destination. When the obstacle is another pedestrian, this switching of goals takes place at the end of each step regardless of the magnitude of $d_{2}$, because the distance changes at each step. These parameters are illustrated in Fig. 12.

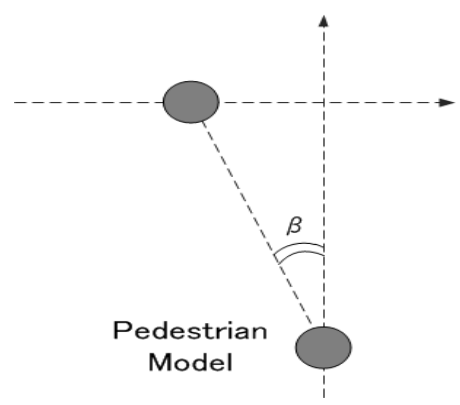

Fig. 13. Definition of $\beta$.

A sub-goal is set as follows:

STEP-1: Select the objects within the range of angle $\alpha$ and distance $r$.

STEP-2: From these selected objects, choose the one with the lowest value of $d_{1}$ as the obstacle to be avoided.

STEP-3: Judge whether $\theta$ is positive or negative. If $\theta$ is positive, set a sub-goal at a distance of $d_{2}$ to the left of the obstacle, and if $\theta$ is negative, set a sub-goal at a distance of $d_{2}$ to the right of the obstacle.

STEP-4: Determine whether the obstacle is another pedestrian or a fixed object. If it is a pedestrian, change the goal from the sub-goal back to the original destination at the end of the step. If it is an object, change the pedestrian's goal back to the original destination if $d_{2}>d_{3}$.

\subsection{Introducing a New Virtual Force to Implement Deceleration Processing}

To solve the problem whereby pedestrians on intersecting paths are unable to avoid each other, we reproduce the decelerating motion that pedestrians perform in consideration of other people. In the proposed model, the psychological effect of consideration to other pedestrians is represented by an external force defined as follows:

$$
f^{a}=-\frac{v-V}{\tau} e^{-(x-d)}
$$

Here, $(v-V) / t$ is a term based on the current speed $\boldsymbol{v}$ and the speed after deceleration $V$, and the maximum value of the external force $f_{a}$ is adjusted by parameter $\tau$. In other words, as the speed decreases, the maximum value of the external force becomes smaller. On the other hand, in the term $e^{-(x-d)}$, where $x$ is the distance to the other pedestrian and $d$ is the distance when the pedestrians pass each other, the external force approaches its maximum value if the distance between the pedestrians is small, but decreases asymptotically to zero if the distance is large. With the addition of this external force $f^{a}$ as a fourth external force term, the model is able to take other pedestrians into consideration.

To allow this force $f^{a}$ to act, we define the angle $\alpha$ as being positive if the other pedestrian is situated in a clockwise direction relative to the direction of travel, and we determine the force as follows: 
STEP-1: Select a pedestrian for whom the value of angle $\alpha$ is no larger than 45 degree

STEP-2: Judge whether this pedestrian will pass in front or behind. If behind, then go to STEP-4.

STEP-3: If $\alpha$ is 7.7 degree or more, calculate $F_{a}$. Otherwise go to STEP-4.

STEP-4: To ensure that $f^{a}$ is an accelerating force, set $f^{a}=2 F^{g}$.

\section{Evaluation Experiment}

\subsection{Overview of Simulator}

\subsubsection{Implementation}

The simulator was developed by Java, using JOGL (Java binding for the OpenGL API) for 3D drawing, and a GUI implemented in Swing. The simulator process is illustrated in Fig. 14. The simulations were run in an event-driven manner with JOGL events handled by three methods and event listeners in the GLEventListener interface, and action listeners defined in Swing.

- init method: A method called only once at the start of a simulation to set up drawing parameters such as light source parameters and reflection parameters for the drawing objects.

- reshape method: A method called to reset the viewpoint position and field of view when the OpenGL drawing canvas is resized.

- display method: A method called when drawing. This method is called repeatedly after the simulation has started. This method is where the action and drawing parts of the agent discussed in Section 3.1 are performed.

- JOGL event listeners: Event listeners defined on the OpenGL drawing canvas.

- Swing action listeners: Action listeners of windows created by Swing and their member objects.

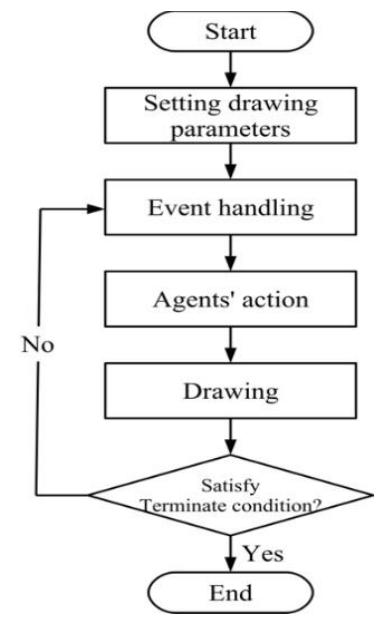

Fig. 14. Simulation flowchart.

\subsubsection{Model implementation}

GUI implementation

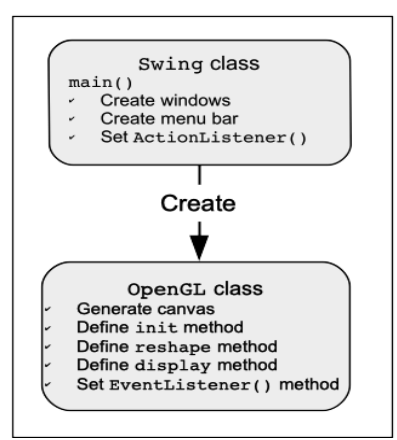

MAS implementation

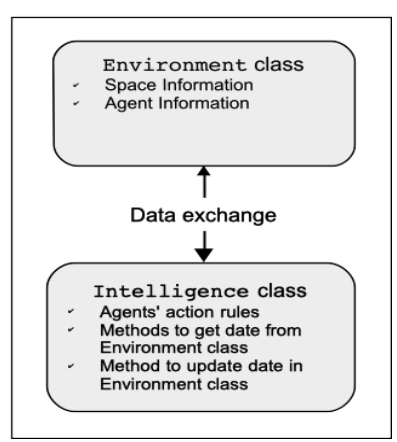

Fig. 15. Simulator configuration. 
The space and agents that make up the MAS are defined as Environment and Intelligence classes. The user first defines all the constituent elements of the space in the Environment class. In the Intelligence class, which defines the action rules governing the behavior of agents, the agent behavior is determined by receiving information from the Environment class. Parts that are changed by the actions of agents are reflected in the Environment class to build relationships between the agents and the space.

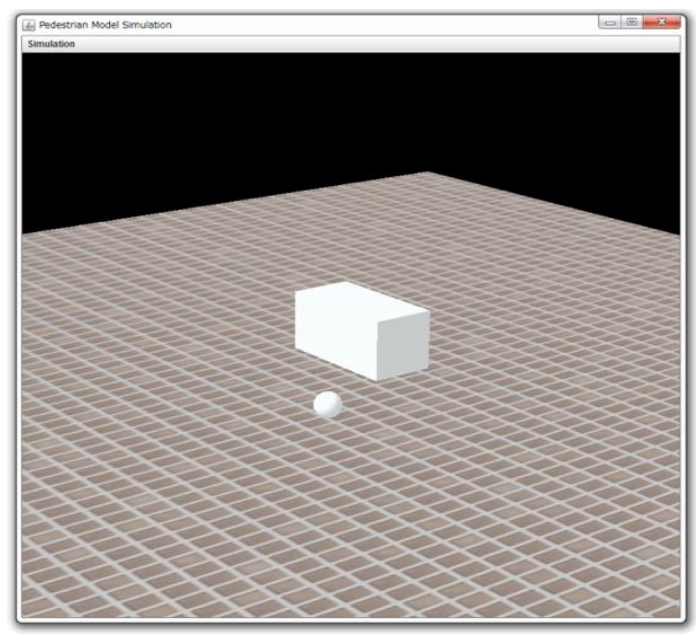

Fig. 16. Simulator screenshot.

\subsection{Sub-goal Experiments}

Sub-goal experiments were performed with the same initial positions as the model verification tests of obstacle avoidance performed in Section 3:

(a) avoiding an obstacle on the walking path $(x=0)$, and

(b) avoiding an obstacle close to the walking path $(x=0.3)$

However, this time the pedestrian parameter $\tau$ was set to 0.01 . This was because the actions performed when switching the goal from the sub-goal to the destination resulted in a slow reaction at the previous value of $\tau=0.1$. The parameters used for sub-goal setting were $r=7.8 \mathrm{~m}, d_{2}=1.8 \mathrm{~m}$, and $\alpha=10$ degree. These parameters were set with reference to the values discussed in Section 3 - avoidance start distance: $7.3 \mathrm{~m}$, distance from obstacle when passing: $41.3 \mathrm{~m}$, angle subtended by obstacle: 10 degree.

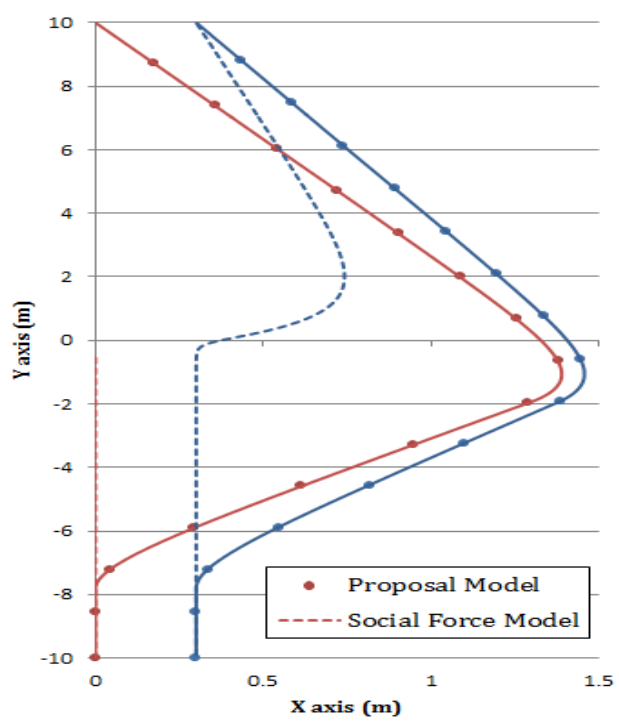

Fig. 17. Avoidance paths. 
The verification results are shown in Fig. 17 (avoidance path) and 18 (speed variation). The avoidance start distance and the distance from the obstacle when passing are summarized in Table 1.

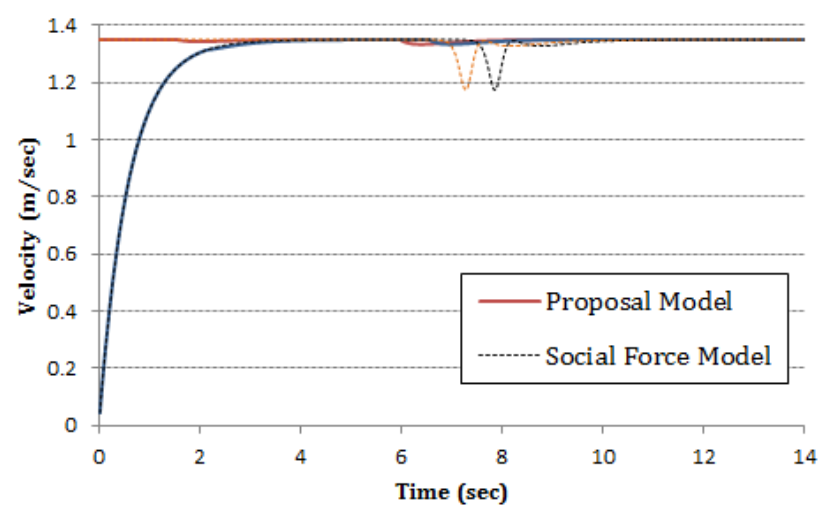

Fig. 18. Speed variation.

Table 1. Distances Representing the Avoidance Characteristics of the Proposed Model

\begin{tabular}{ccc}
\hline \hline Initial Condition & Distance at start of avoidance & Distance from obstacle when passing \\
\hline (a) & $7.5 m$ & $1.33 m$ \\
(b) & $7.5 m$ & $1.40 m$ \\
\hline \hline
\end{tabular}

\subsection{Deceleration Processing Experiment}

The deceleration processing verification experiments were performed under the following initial conditions:

(a) the same conditions as the intersecting path avoidance experiment of Section 3

(b) the conditions of Fig. 19, under which deceleration processing was started in a previous study

In experiments performed with deceleration processing parameters of $V=0.7 \mathrm{~m} / \mathrm{s}$ and $\mathrm{d}=1.4 \mathrm{~m}$, the avoidance paths and speed variation for case (a) are shown in Fig. 20 and 21, respectively. Fig. 22 shows the speed variation for case (b).

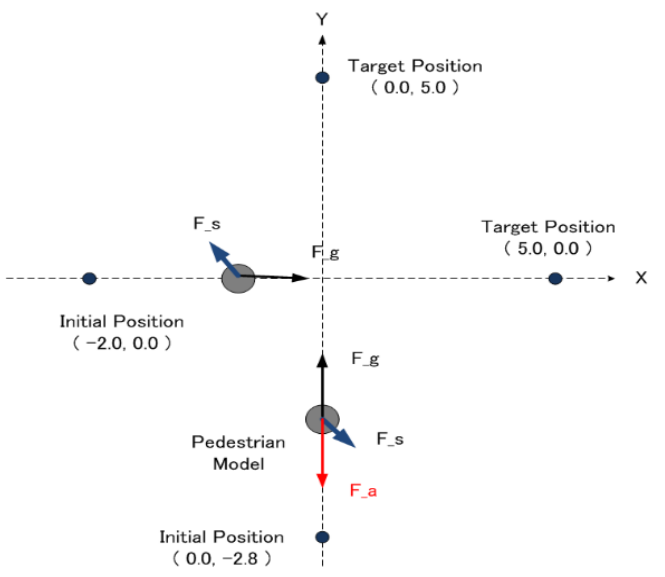

Fig. 19. Initial positions in case (b).

As Fig. 22 shows, the pedestrians were able to pass each other under the starting conditions of case (a). In this case, the distance between the pedestrians while passing was $0.91 \mathrm{~m}$. At $2.3 \mathrm{~s}$ after the start of strong deceleration, the minimum speed of $0.72 \mathrm{~m} / \mathrm{s}$ is reached, after which the pedestrian accelerates back to 
normal speed after $1.14 \mathrm{~s}$. On the other hand, Fig. 22 shows the speed variation when simulated under the conditions of case (b). The distance from the other pedestrian at the time of passing was $1.39 \mathrm{~m}$. After $1.19 \mathrm{~s}$ of strong deceleration, a minimum speed of $0.8 \mathrm{~m} / \mathrm{s}$ was reached, and then the pedestrian accelerated back to a normal speed after $0.93 \mathrm{~s}$.

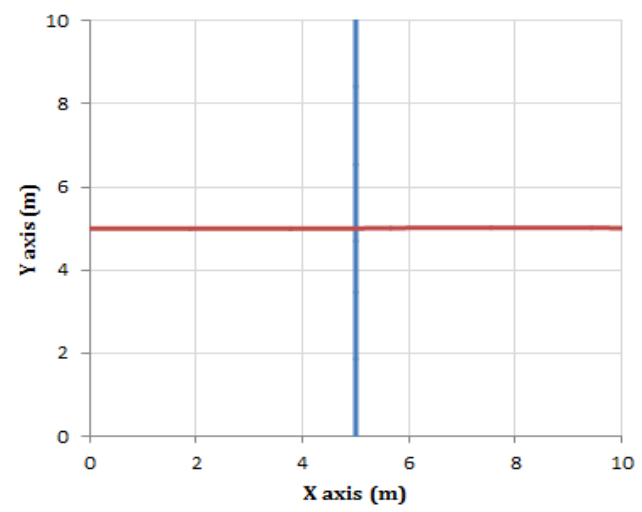

Fig. 20. Pedestrian paths.

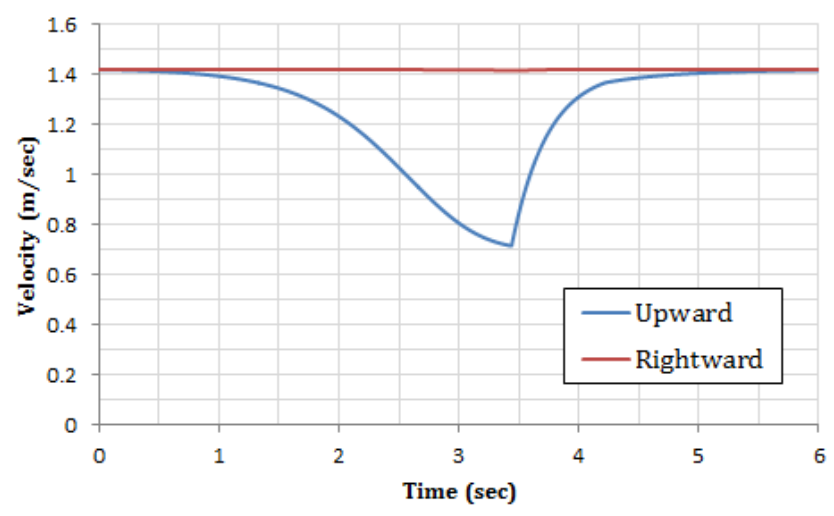

Fig. 21. Speed variation of case (a).

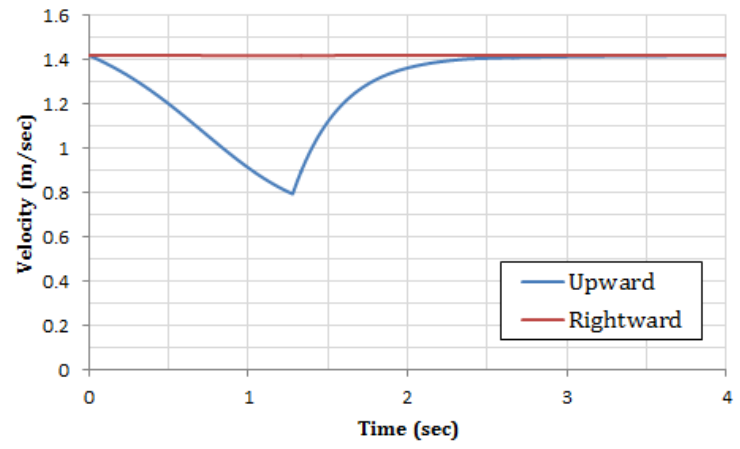

Fig. 22. Speed variation of case $(b)$.

\section{Discussion}

\subsection{Sub-goal Setting}

With the proposed model, it was possible to avoid obstacles present on the pedestrian's walking path, and in the vicinity of the walking path. Also, regarding the distance to the obstacle at the time when avoidance is started, which is a pedestrian avoidance characteristic discussed in Section 3, we obtained a value of $7.5 \mathrm{~m}$. 
This is close to the target value of $7.34 \mathrm{~m}$ and demonstrates the validity of the sub-goal parameter $r$. We were also able to keep the distance from the obstacle while passing within the target range of $0.7-1.3 \mathrm{~m}$, demonstrating the validity of parameter $d_{2}$. With regard to the issues of a pedestrian's path after passing an obstacle, we successfully suppressed the deceleration effect by setting parameter $d_{3}$ so as to change the destination before reaching the sub-goal.

Focusing on the speed variation, a deceleration of about $15 \%$ was observed in the conventional model, but by setting a sub-goal we were able to suppress the deceleration to $5 \%$. This means that setting a sub-goal made it possible to reproduce the characteristic whereby pedestrians move constant speed regardless of any avoidance paths.

From the above results, it can be said that we were successful in reproducing the obstacle avoidance behavior of pedestrians, which was the original purpose of introducing sub-goals. However, generic simulators must be able to cope with more complex conditions such as multiple obstacles. Here, we consider the behavior of the model shown in Fig. 23 in order to consider the issues of the proposed model.

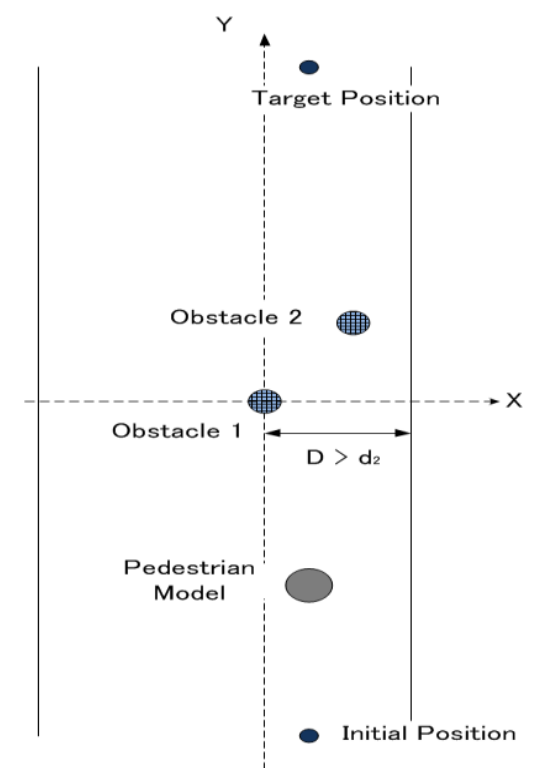

Fig. 23. A problematic initial arrangement of obstacle.

In our proposed model, obstacle $\mathbf{1}$ is selected as the obstacle to be avoided, and based on the positional relationship of the pedestrian and the obstacle, is situated in such a way that a sub-goal is positioned at a distance $d_{2}$ to the right of the obstacle so as to minimize the distance between the pedestrian and the destination. However, when obstacle 2 lies beyond obstacle 1, a pedestrian might choose to go to the left of obstacle 1. The inability of the proposed model to make such judgments is an issue worthy of further study. In the future, the model will need to incorporate a decision unit that performs this sort of path selection.

\subsection{Deceleration Processing}

We were able to avoid another pedestrian in the two verification experiments and were able to show that the new forces added to the proposed model are effective for deceleration processing. Furthermore, with regard to the variation of speed during avoidance, we found that the minimum speeds after deceleration in the verification experiments $-0.72 \mathrm{~m} / \mathrm{s}$ in (a) and $0.8 \mathrm{~m} / \mathrm{s}$ in (b) - were similar to the speed of $0.8 \mathrm{~m} / \mathrm{s}$ reported in the literature. This also demonstrates the validity of the minimum speed $V$ as a deceleration parameter.

However, in experiments with two pedestrians passing in opposite directions, the distance between then was $0.91 \mathrm{~m}$ in (a) and $1.39 \mathrm{~m}$ in (b), which is slightly different from the value of $1.5 \mathrm{~m}$ reported in the literature, 
and the acceleration and deceleration times measured in (a) were longer than the values reported in the literature. This means there is room for improvement in the proposed model. Perhaps the reduced distance from the other pedestrian in these experiments was caused by the longer deceleration time. The longer deceleration time may have been caused by the force $F^{a}$ increasing as the two pedestrians approached each other but decreasing when this distance became smaller than the passing distance $d$ set as a parameter. However, this distance is a value determined based on time and speed factors, so a simple reduction of the deceleration time will not lead to a solution for this problem. In the future, it will therefore be necessary to solve this problem by understanding the characteristics of pedestrians during deceleration in greater detail.

\subsection{Future Works and Challenges}

One of the challenges to advancement of this research is the scarcity of data relating to pedestrian characteristics. In particular, since there have hardly been any studies made of deceleration movements, it will be necessary to clarify the characteristics of these movements by conducting experiments with actual pedestrians. It should also be noted that the validity of the model proposed in this article has only been tested in experiments with very simple environments with a basic set-up consisting of one pedestrian and one obstacle, or two pedestrians and no obstacles. The validity of this model's behavior should therefore be tested with a wider range of initial arrangements.

\section{Conclusion}

In this article, in order to address the issues of the existing Social Force Model (SFM), we have proposed a new model that incorporates sub-goals and deceleration movements. We evaluated this model and confirmed its validity by conducting obstacle avoidance and pedestrian avoidance simulations. As a result, we were able to reproduce the avoidance behavior used in path selection by introducing sub-goals and were able to reproduce pedestrian avoidance behavior that cannot be achieved with SFM, even with deceleration movements.

\section{Acknowledgment}

This work is supported by JSPS Grant-in-Aid for Scientific Research (KAKENHI) (C) Grant Number JP18K04623.

\section{References}

[1] Kaneda, T. (2010). Pedestrian Agent Simulation by using Artisoc. Tokyo: Kozo-Keikaku Engineering, Inc. Publishers.

[2] Helbing, D., \& Molnar, P. (1995). Social force model for pedestrian dynamics. Physical Review E, 51, 42824286.

[3] Helbing, D., Farkas, I. J., Molnar, P., \& Vicsek, T. (2002). Simulation of pedestrian crowds in normal and evacuation situations. In M. Schreckenberg, and S. D. Shama (Eds.), Pedestrian and Evacuation Dynamics (pp. 21-58). London: Springer.

[4] Tatebe, K., Tsujimoto, M., \& Shida, K. (1994). Methods for judging the beginning point of avoiding behavior and avoidance distance between a pedestrian and a standing obstacle: A study on pedestrian behavior of avoiding obstacles (II) Journal of Architecture, Planning and Environmental Engineering, Architectural Institute of Japan, no. 465, pp. 95-104.

[5] Yoda, M., \& Shiota, Y. (1998). Motion characteristics and avoidance motion algorithm based on human passing behavior: Results of experiments under natural and laboratory settings [in Japanese]. Transactions of the Japan Society of Mechanical Engineers, 64(619), pp. 959-965. 
[6] Yamamoto, T., Shibata, S., \& Jindai, M. (2006). An analysis of human avoidance behaviour of a collision by deceleration in crossing each other. Japan Ergonomics Society, 42(6), pp. 402-407.

[7] Yanagisawa, Y., Yamada, T., Hirata, K., \& Satoh, T. (2006). Pedestrian dynamics with decision of Subgoals based on eye-gaze. IPSJ Journal, 47(7), 2160-2167.

Tohru Mizuno received his B.Eng and M.Eng in information and computer science from Doshisha University in 2012 and 2015 respectively. He also received the Diplome d'Ingenieur (French engineering degree) from Ecole Centrale du Lille, Lille, France in 2014. After finished his master's study, he joined to Hitachi, Ltd.. Currently, he is an engineer of System Control Engineering Division of Hitachi, Ltd.

Hirohide Haga received his B.Eng and M.Eng in electronics from Doshisha University and Ph.D. in computer science from Kyoto University in 1978, 1980, and 1996 respectively.

After finishing his master's study in 1980, he joined in Hitachi, Ltd., where he was a research staff member from 1980 to 1994. In 1994, he moved to Doshisha University as a faculty member. Currently, he is a full professor of computer and information science at Doshisha University, Kyoto, Japan.

Prof. Haga is a member of IEEE-CS, ACM, BCS (British Computer Society), and IEICE-J (Institute of Electronics, Information and Computer Engineers of Japan). He held several visiting positions (visiting scholar, visiting professor, and invited professor) at Imperial College of Science and Technology, University of London (UK), University of Oulu (Finland), Cambridge University (UK), École Centrale de Lille (France), and CentraleSupélec (France). His research interests include software engineering, multi-agent simulation, blockchain application, and digital art. He was honored as a Chartered IT Professional (CITP) from BCS. 